\title{
Prevalencia de Eventos Cardiocerebrovasculares en Pacientes con Cáncer de Próstata en Manejo con Bloqueo Androgénico con Análogo de GNRH
}

\section{Prevalence of Cardiovascular Events in Patients with Prostate Cancer that Require Androgen Deprivation Therapy with Gonadotroping Hormone-Releasing Hormone Agonists}

\author{
José Luis Poveda ${ }^{1}$ Cesar González ${ }^{1} \quad$ William Quiroga ${ }^{1} \quad$ Margarita Zapata ${ }^{1}$ Sebastián Baena ${ }^{1}$ \\ ${ }^{1}$ Departamento de Urología, Hospital Central de la Policía y Hospital \\ Militar Central, Bogotá, Colombia \\ Address for correspondence José Luis Poveda, MD, Departamento de \\ Urología, Hospital Central de la Policía y Hospital Militar Central, \\ Calle 134 n 7b 83, Consultorio 1007, Bogotá, Colombia \\ Urol Colomb 2018;27:48-54 \\ (e-mail: povedamatiz@gmail.com).
}

\section{Resumen \\ Palabras Clave \\ - cáncer de próstata \\ - prevalencia \\ - análogos GNRH \\ - eventos cardiocere- brovasculares \\ - ACE27 \\ - estudio descriptivo}

Objetivo Determinar la prevalencia de eventos cardiocerebrovasculares en pacientes con cáncer de próstata que son manejados con bloqueo hormonal, con análogo de la GNRH. Materiales y Métodos Se realizó un estudio de corte transversal, en pacientes con cáncer de próstata que fueron manejados con bloqueo hormonal con análogo de la GNRH, con un mínimo de seguimiento de 2 años, entre los años 2004 y 2014. Paciente con eventos que acaecieron en el primer año de observación, fueron excluidos. Se evaluaron las comorbilidades de los pacientes y se clasificaron en diferentes grupos de riesgo, según el índice de comorbilidades ACE27. Se determinó la prevalencia de eventos cardiovasculares definidos como un episodio de infarto agudo de miocardio, de angina y de eventos cerebrovasculares durante la observación. Se comparó la prevalencia de acuerdo al subgrupo de riesgo ACE27.

Resultados Se incluyeron 281 pacientes. La comorbilidad más común fue la hipertensión arterial con un $45 \%$ y por último la diabetes mellitus con un $17 \%$. La prevalencia de eventos cardiovasculares y cerebrovasculares en la población general fue del 7,5\% para cada evento. Se clasificaron los pacientes según el índice de comorbilidades ACE- 27 tendiendo el 39\% de los pacientes un índice de comorbilidad bajo y el $7 \%$ alto. La prevalencia de eventos cardiocerebrovasculares varía desde el $5 \%$ en bajo riesgo, hasta el $25 \%$ en grupo de alto riesgo.

Conclusiones La prevalencia de eventos cardiocerebrovasculares fue del 7,5\% en la población de estudio. En el grupo de alto riesgo ACE27 con el uso de análogo de GNRH, se determinó una mayor prevalencia de eventos cardiocerebrovasculares.
Objective To determine the prevalence of cardiovascular events in patients with prostate cancer who were being managed androgen deprivation therapy with gonadotroping hormone-releasing hormone (GHRH) agonists. received

March 24, 2017

accepted

July 19, 2017

published online

March 13, 2018
DOI https://doi.org/

10.1055/s-0038-1637015

ISSN 0120-789X.

eISSN 2027-0119.
Copyright $\odot$ 2018, Sociedad Colombiana License terms de Urología. Publicado por Thieme Revinter Publicações Ltda., Rio de Janeiro, Brazil. Todos los derechos reservados. (c) (i) $\ominus$ (\$) 


\section{Keywords}

- prostate cáncer

- prevalence

- luteinizing hormonereleasing hormone (LHRH) agonists

- ACE27

- descriptive study
Materials and Methods A cross-sectional analysis was done in patients with prostate cancer that were treated with androgen deprivation therapy with GHRH agonists, between 2004 and 2014. Follow-up of 2 years was required. Patients who developed cerebrovascular and cardiovascular events within the first year of treatment were excluded. Comorbidities were evaluated and classified in groups of risk, according to the adult comorbidity evaluation index (ACE27). General prevalence of cardiovascular events was established. Prevalence between comorbidity risk groups were quantified and compared.

Results 281 patients were included. Among all comorbidities arterial hypertension was the most frequent (45\%) and the less frequent was diabetes mellitus (17\%). The prevalence of cardiovascular and cerebrovascular events in general population was 7.5\%. Patients were classified according to the ACE27 index score resulting in 39\% of patients having mild comorbidity score and $7 \%$ as severe comorbidity. Between patients classified as having mild comorbidity, prevalence of $5 \%$ was found and $25 \%$ of prevalence in the severe comorbidity group.

Conclusion The prevalence of cardiovascular events was $7.5 \%$ in the studied population. In the severe risk group, according to the ACE 27 index, the prevalence of cardiovascular events increases till $25 \%$.

\section{Introducción}

Actualmente, la terapia con bloqueo androgénico (BA), con agonistas de la hormona liberadora de GnRh y/o bloqueadores de los receptores androgénicos, son junto a la orquidectomía, las alternativas disponibles de supresión hormonal, de mayor uso en cáncer de próstata local avanzado y metastásico, habiendo aumentado su frecuencia de uso como primera opción. ${ }^{1,2}$

Los niveles bajos de testosterona, consecuencia del BA, se comportan como un factor de riesgo independiente para el desarrollo de un síndrome metabólico. Diversos estudios han demostrado la asociación entre el síndrome metabólico y un aumento del riesgo de enfermedad cardiovascular. ${ }^{3}$ Una revisión reciente concluyó que el riesgo de mortalidad cardiovascular se incrementó un $17 \%$ en los pacientes tratados con bloqueo hormonal. ${ }^{4}$ De igual manera, se encuentra evidencia contradictoria entre el uso de agonistas de la GnRh específicamente y el aumento de eventos cardiovasculares. ${ }^{5}$ Todo ello ha conducido a que diversas sociedades científicas elaboren recomendaciones sobre la necesidad de valorar el incremento del riesgo que se produce en esas circunstancias. ${ }^{6}$

El objetivo principal de este estudio es determinar la prevalencia de eventos cardiocerebrovasculares en pacientes con cáncer de próstata que son tratados con bloqueo androgénico con agonistas de la GNRH. En forma secundaria se determina la prevalencia de eventos cardiocerebrovasculares estratificados por grupo de riesgo, de acuerdo al índice de comorbilidades del adulto ACE-27.

\section{Metodología}

Se diseñó un estudio de corte transversal, para determinar prevalencia. Se determinó como población de estudio; todos los pacientes con cáncer de próstata local avanzado y metastásico, que fueron manejados con bloqueo hormonal a través del uso de agonista de la GNRH, en el Hospital Central de la Policía y en el Hospital Militar Central durante los últimos 10 años. Dicho manejo fue seleccionado por medio del criterio del médico tratante.

La información fue obtenida a través de las bases de datos de diagnósticos de consulta externa (RIPS), suministro de medicamentos y registros de historia clínica de los hospitales.

Se reclutaron los pacientes de forma probabilística y sistemática, con inicio temporal a partir del año 2004.

Los pacientes incluidos debían tener el inicio de tratamiento entre enero de 2004 y diciembre de 2014 , debían contar con un mínimo de seguimiento de 2 años y en todos los casos las consultas y los paraclínicos de seguimiento se realizan en la misma institución por el tipo de aseguramiento en salud. No se discrimina por subtipo de agonista de la GnRh. Fueron excluidos pacientes con antecedente de evento cardiovascular o cerebrovascular en el primer año de seguimiento.

Se estudiaron las variables edad, comorbilidades más relevantes como hipertensión arterial, dislipidemia, diabetes mellitus y arritmias cardíacas. También se documentó la fecha de inicio del bloqueo hormonal y la continuidad del mismo (intermitencia o no). Posteriormente se realiza una estratificación del grupo, según el índice de comorbilidades del adulto ACE27. Se describe el número de eventos cardiocerebrovasculares registrados en el periodo de seguimiento para el grupo general. Finalmente se describen los eventos cardiocerebrovasculares de acuerdo a la clasificación del riesgo cardiovascular según el índice de comorbilidades del adulto ACE27.

La información es procesada con el programa estadístico STATA 12 (StataCorp®). Se realiza la estandarización de las variables y los análisis descriptivos de las diferentes variables. En el caso de variables continuas se utilizan 
medidas de tendencia central y de dispersión. Para las variables categóricas, se utilizan medidas de proporción. Se determina la prevalencia de eventos cardiocerebrovasculares en el grupo general.

Después se realizaron análisis bivariados; para describir la prevalencia de las diferentes variables en los subgrupos inicialmente definidos. Se realizó una aproximación a la significancia estadística, donde en las variables continuas se utilizó la prueba U Mann Whitney y para las variables categóricas, la prueba chi2 o el test de Fisher. Se definieron cuatro grupos de riesgo según el índice ACE27 para la comparación entre ellos, respecto a la prevalencia de eventos cardiocerebrovasculares, se realiza un análisis de varianza no paramétrica.

Posteriormente se realizó un análisis de oportunidad relativa (OR), para estimar la asociación entre eventos cardiocerebrovasculares y los grupos de riesgos establecidos. Finalmente se estimó la razón entre las proporciones de prevalencia de la enfermedad.

Se describen las respectivas medidas de precisión y azar para cada valor. Se contempló en este estudio, resultados estadísticamente significativos con valores de p menores a 0,05.

\section{Resultados}

Posterior a la revisión de las historias clínicas y bases de datos, de acuerdo al procedimiento preestablecido y con referencia a los criterios de inclusión y de exclusión, se incluyeron finalmente los 281 pacientes.

Las características de la población se muestran en la - Tabla 1.

En este caso, se identifica un grupo de pacientes con un promedio de edad alto, que como es esperable tiene diversos factores de riesgo asociados a eventos cardiocerebrovasculares. El factor de riesgo con mayor frecuencia fue hipertensión arterial.

Los pacientes fueron clasificados de acuerdo al grupo de riesgo con la escala ACE27, encontrando una mayor frecuencia de pacientes entre el grupo de menor riesgo. En la - Tabla 2 se muestra la distribución de los subgrupos.

La prevalencia general de eventos cardiocerebrovasculares en el grupo estudiado fue de 21 casos, equivalente al 7,5\%. Se muestra su representación en la - Fig. $\mathbf{1}$.

Al comparar la distribución de la prevalencia de eventos de acuerdo al grupo de riesgo ACE27, se observa en la - Tabla 3.

No se encuentran casos de eventos en el grupo 0 del índice ACE27. Se observa un aumento progresivo de la prevalencia,

Tabla 1 Descripción de la población

\begin{tabular}{|l|l|}
\hline Variable & Valor medio \\
\hline Edad & 76.5 años \\
\hline Bloqueo androgénico intermitente & $62 \%$ \\
\hline Arritmias & $9.60 \%$ \\
\hline HTA & $45 \%$ \\
\hline Diabetes mellitus & $17 \%$ \\
\hline Dislipidemia & $23 \%$ \\
\hline
\end{tabular}

Tabla 2 Distribución del riesgo de acuerdo al índice ACE27

\begin{tabular}{|l|l|l|}
\hline IÍndice ACE27 & Frecuencia & Porcentaje \\
\hline 0 & 46 & 16.36 \\
\hline 1 & 110 & $39.15 \%$ \\
\hline 2 & 105 & $37.37 \%$ \\
\hline 3 & 20 & $7.12 \%$ \\
\hline
\end{tabular}

Abreviaciones: ACE27, índice de comorbilidades del adulto.

según el grupo de riesgo, alcanzando un 25\% de eventos dentro del grupo 3 o de alto riesgo.

Se nota una progresión de la prevalencia respecto al grupo de riesgo ACE27, particularmente en el grupo 3 de alto riesgo, como se muestra en la - Fig. 2.

Posterior a la valoración de la prevalencia, se comparan los valores de prevalencia de eventos respecto al grupo de riesgo ACE27. Por estadística no paramétrica, se realiza un análisis de varianza Kruskal Wallis y se muestra en la - Tabla 4.

Considerando que no existe una diferencia estadísticamente significativa entre los grupos de riesgo y la ocurrencia de los eventos cardiocerebrovasculares, este dato puede ser atribuible a la distribución y tamaño muestral. Clínicamente es evidente una mayor prevalencia de eventos dentro del grupo 3 de riesgo ACE27.

Respecto al cálculo de las razones de prevalencia, no se presentaron eventos cardiocerebrovasculares entre el grupo 0 del índice ACE27, siendo ese el grupo comparativo ideal. En forma consecuente, las prevalencias de los grupos 1, 2 y 3 no son comparables con el grupo 0 o con la población sin factores de riesgo.

Comparando el subgrupo 3, con mayor riesgo y prevalencia de eventos respecto al grupo 1, se construye la - Tabla 5.

Se encontró una razón de 4,58 eventos en el grupo 3 por cada uno del grupo 1. Se logra la significancia estadística, pero con una precisión marginal. Posiblemente atribuible a las características muestrales.

Comparando el subgrupo 3, con mayor riesgo y prevalencia de eventos respecto al grupo 2, se construye la - Tabla 6 .

En ese caso se identifica una razón de 2,65 eventos en el grupo 3 respecto a 1 caso del grupo 2 . No se alcanza una diferencia estadísticamente significativa y la precisión es equívoca nuevamente.

Las diferentes herramientas estadísticas muestran una tendencia a encontrar mayores eventos cardiocerebrovasculares dentro del grupo 3 como se puede ver con el análisis OR. Aunque los valores no tienen una significancia estadística, los ya encontrados indican una mayor atención clínica de ese grupo de pacientes por la frecuencia de ese tipo de eventos durante el uso de análogos de GnRH.

\section{Discusión}

Para la Organización Mundial de la Salud, las enfermedades más frecuentes asociadas con la mortalidad en el mundo, son las cardiovasculares. Dentro de esas enfermedades se 


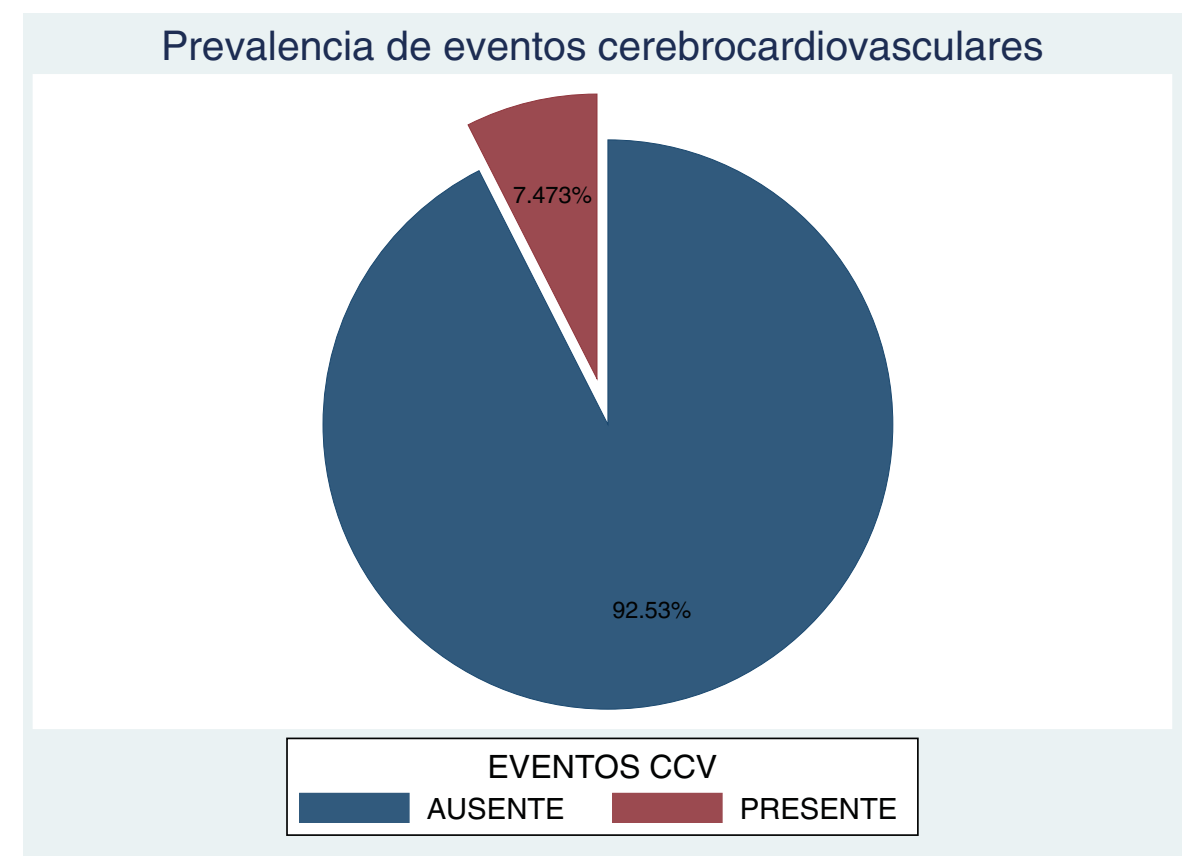

Fig. 1 Prevalencia de eventos cardiocerebrovasculares (CCV).

destacan como factores de riesgo la hipertensión arterial (13\% de las muertes a nivel mundial), el tabaquismo (9\%), la diabetes mellitus (6\%), el sedentarismo (6\%), el sobrepeso y la obesidad $(5 \%){ }^{7}$ De igual manera se destaca la prevalencia existente del cáncer de próstata, siendo la enfermedad maligna más frecuente entre hombres. ${ }^{8}$ Adicionalmente es importante indicar la alta prevalencia de estadios localmente avanzados y metastásicos del cáncer de próstata en los países en desarrollo ${ }^{9}$ y que posiblemente serán manejados con terapia de supresión hormonal.

En la literatura disponible sobre el manejo de supresión hormonal del cáncer de próstata, diversos trabajos han establecido una relación entre el uso de análogos de la GnRh y el desarrollo de eventos cardiocerebrovasculares. En abril de 2014, un estudio observacional danés mostró la asociación entre el uso de análogos de la GnRh y un aumento en el riesgo de infarto agudo de miocardio (IAM) o de derrame cerebral en una población de pacientes con cáncer de próstata. Sin embargo, ese evento adverso no se encontró entre los pacientes la castración quirúrgica. ${ }^{10}$ Previamente, otro estudio de vigilancia con la base de datos de Epidemiología y

Tabla 3 Prevalencia de eventos respecto al índice ACE27

\begin{tabular}{|l|l|l|}
\hline \multicolumn{3}{|l|}{ Frecuencia de eventos respecto al grupo de riesgo } \\
\hline Grupo ACE27 & Eventos & Porcentaje \\
\hline 0 & 0 & $0 \%$ \\
\hline 1 & 6 & $5.45 \%$ \\
\hline 2 & 10 & $9.52 \%$ \\
\hline 3 & 5 & $25 \%$ \\
\hline
\end{tabular}

Abreviaciones: ACE27, índice de comorbilidades del adulto.
Resultados Finales (SEER) -Medicare, también encontró una relación estadísticamente significativa entre el uso de análogos de la GnRH, la enfermedad coronaria aguda, infartos de miocardio y la muerte súbita cardiogénica $(p<0,001)$, pero no con la orquiectomía ( $\left.\mathrm{p}^{3} 0,7\right) .{ }^{11}$ Esos estudios observacionales, añadieron una nueva evidencia al trabajo publicado por primera vez por Keating y col. en 2006, en el que se demostró un aumento del riesgo de infarto agudo de miocardio con el uso de análogos de GnRh y la ausencia de dicho riesgo con la orquiectomía simple bilateral. ${ }^{2}$

Otras investigaciones adicionales, también han sugerido que el riesgo de hipertensión arterial, diabetes, derrames cerebrales, infartos cardiacos, e incluso la muerte a causa de enfermedad cardíaca es mayor en hombres que son tratados con terapia de supresión hormonal por diferentes mecanismos. Varios estudios han mostrado como el uso de análogos de la GnRH se asocia con el aumento de la grasa corporal total, disminución de la sensibilidad de la insulina, diabetes mellitus, y con el aumento del colesterol tipo LDL, lo cual se asocia finalmente con un aumento de la enfermedad cardiovascular. $^{2,12}$ Igualmente, se ha encontrado relación entre el uso análogos de la GnRh y un mayor riesgo de eventos tromboembólicos. ${ }^{1}$ Saigal y col. reportaron que, hombres diagnosticados recientemente con cáncer de próstata, que recibieron bloqueo hormonal durante al menos un año, tienen un $20 \%$ de riesgo más alto de mortalidad cardiovascular, comparados con los pacientes que no recibieron la terapia de deprivación androgénica. ${ }^{4}$

Una revisión sistemática presentada por Carneiro y col., en 2015, estableció también la asociación entre eventos cardiovasculares y el uso de análogos de GnRh. La conclusión hace referencia al impacto en la morbilidad cardiovascular de esos medicamentos, con el consiguiente impacto en la calidad de vida. ${ }^{13}$ 


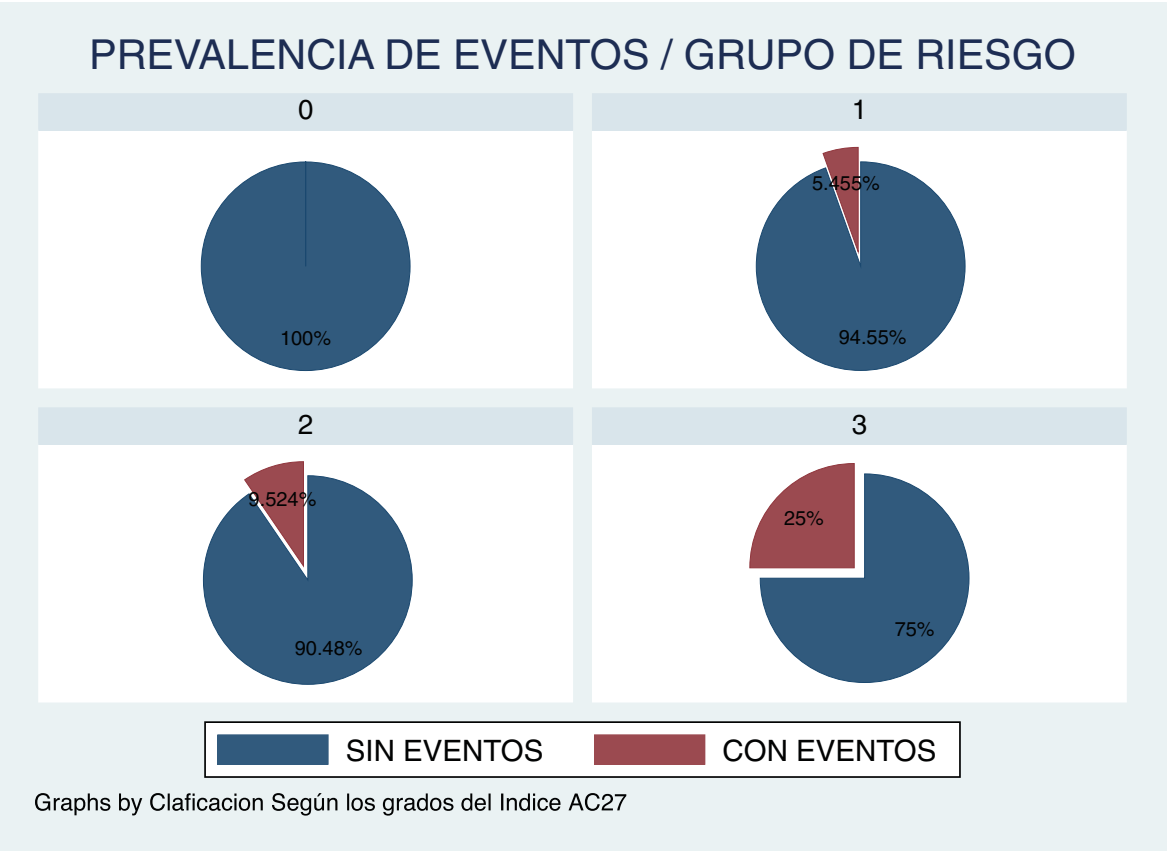

Fig. 2 Prevalencia de eventos cardiocerebrovasculares según los grupos de riesgo.

Tabla 4 Análisis de varianza Kruskal Wallis

\begin{tabular}{|c|c|c|}
\hline \multicolumn{3}{|c|}{$\begin{array}{l}\text { Análisis de varianza desenlace eventos respecto a grupos } \\
\text { de riesgo }\end{array}$} \\
\hline Grupo & Observaciones & Rank SUM \\
\hline 0 & 46 & 6003 \\
\hline 1 & 110 & 15198 \\
\hline 2 & 105 & 15107.5 \\
\hline 3 & 20 & 3312.5 \\
\hline Chi-squared= & 2.871 & 3D.F. \\
\hline Probability & 0.412 & \\
\hline With ties $=$ & 13.837 & 3D.F. \\
\hline Probabilidad & 0.031 & \\
\hline
\end{tabular}

Abreviaciones: DE, degrees free.
A pesar de toda esa literatura disponible, otros trabajos no han demostrado asociación entre el uso de análogos de GnRh y el desarrollo de eventos cardiovasculares. ${ }^{14}$ Incluso se discute la metodología empleada en varios de los trabajos que establecen la asociación. ${ }^{5}$ Entre ellos, un metanálisis publicado en JAMA, no logró demostrar la asociación entre el uso de los agonistas de la GnRh y la mortalidad cardiovascular. ${ }^{12}$

Esos hallazgos hacen que la evidencia sea contradictoria. Muchos de los trabajos donde se establece la relación entre el uso de análogos y eventos cardiocerebrovasculares, tienen defectos en su metodología epidemiológica y validez interna. En los mejores diseños, no se establece la relación en forma definitiva.

La prevalencia de eventos cardiocerebrovasculares del grupo general en este estudio fue del 7,5\%, que es similar a lo que se reporta en la literatura respecto a la población

Tabla 5 Oportunidad de riesgo en subgrupo 3 con respecto al grupo 1

\begin{tabular}{|l|l|l|l|l|l|l|}
\hline \multicolumn{2}{|l|}{ Razón de prevalencias entre el grupo ACE27 y el grupo 1 } \\
\hline Grupo & Casos & Total & Razón prevalencias & (95\% IC) & OR & (95\% IC) \\
\hline 3 & 5 & 20 & 4.58 & $1.54-13.5$ & 5.77 & $1.65-20.31$ \\
\hline 1 & 6 & 110 & & & & \\
\hline
\end{tabular}

Abreviaciones: ACE27, índice de comorbilidades del adulto; IC, intervalo de confianza; OR, odds ratio.

Tabla 6 Oportunidad de riesgo en subgrupo 3 con respecto al grupo 2

\begin{tabular}{|c|c|c|c|c|c|c|}
\hline \multicolumn{7}{|c|}{ Razón de prevalencias entre el grupo ACE27 3 y el grupo 2} \\
\hline Grupo & Casos & Total & Razón prevalencias & (95\% IC) & OR & (95\% IC) \\
\hline 3 & 5 & 20 & \multirow[t]{2}{*}{2.65} & \multirow[t]{2}{*}{$1.0-6.86$} & \multirow[t]{2}{*}{3.16} & \multirow[t]{2}{*}{$0.99-10.2$} \\
\hline 2 & 10 & 105 & & & & \\
\hline
\end{tabular}

Abreviaciones: ACE27, índice de comorbilidades del adulto; IC, intervalo de confianza; OR, odds ratio. 
general. Por ejemplo, en España en el 2006, se reporta una prevalencia de infarto agudo de miocardio del $6,49 \%$ en pacientes de 65 a 74 años y del $8,87 \%$ en pacientes con más de 75 años, ese grupo específicamente sin cáncer de próstata. ${ }^{15}$ Se puede establecer que la prevalencia encontrada no difiere en mayor medida de la población general, que no estuvo expuesta a la administración de agonistas de la GnRh.

En este estudio, se observó que el $81 \%$ de los hombres incluidos, tenían factores de riesgo cardiovascular al inicio del estudio. Investigaciones anteriores también mostraron que las morbilidades cardiovasculares son comunes en pacientes con cáncer de próstata. El trabajo de Albertsen y col., mostró cómo al menos el 30\% de los pacientes incluidos en experimentos clínicos tenían enfermedades o factores de riesgo cardiovascular al momento del inicio. ${ }^{16}$ Así, el grupo de estudio tiene un perfil de riesgo cardiovascular similar al reportado en otros estudios.

La prevalencia de eventos cardiocerebrovasculares del estudio fue aumentando en forma proporcional al riesgo de comorbilidad según el Índice ACE27, siendo del 5,6\% y un $25 \%$, en el grupo de riesgo bajo y alto, respectivamente. Sin embargo, no se encontró una diferencia estadísticamente significativa entre los grupos de riesgo y la ocurrencia de los eventos cardiocerebrovasculares $(p=0.4)$. Ese dato puede ser atribuible a las características muéstrales. Sin embargo, clínicamente es evidente una mayor prevalencia de eventos dentro del grupo de riesgo alto ACE27. Esos hallazgos se corroboran con otros estudios publicados. En el trabajo de Gandaglia y col., se aplicó el Índice de comorbilidad de Charlson (CCI) a los pacientes y se les clasificó según su comorbilidad. Se demostró que las tasas de mortalidad CV variaron de $3,7 \%$ a $16,3 \%$ para los individuos más jóvenes en el estrato más bajo de $\mathrm{CCI}$, a los individuos de más edad dentro del estrato más alto de CCI. Esa relación sugiere que la mortalidad cardiovascular se ve afectada por la edad y el CCI. En esos análisis se concluye que la morbilidad cardiovascular basal, representa el más fuerte predictor de la mortalidad cardiovascular. ${ }^{16}$ Los resultados de este trabajo están de acuerdo con lo señalado en esos estudios, donde si aumentamos la comorbilidad también se aumenta la prevalencia de eventos cardiocerebrovasculares.

En un experimento clínico que evaluó la eficacia de la radioterapia y la adyuvancia con supresión hormonal, se observó cómo la administración del bloqueo hormonal, aumentó el riesgo de muerte cardiaca, específicamente en pacientes con comorbilidad moderada a severa previa, según el índice ACE27. ${ }^{17}$ En forma similar el trabajo publicado por Nanda y col., mostró que el bloqueo hormonal, aumenta el riesgo de mortalidad cardiovascular en pacientes con una historia de episodios cardíacos o en alto riesgo de enfermedad coronaria. ${ }^{6}$

Los resultados obtenidos de esos estudios, junto con la mayor prevalencia de eventos cardiocerebrovasculares en el grupo de alto riesgo cardiovascular de este trabajo, generan una consideración especial antes de comenzar la terapia de deprivación hormonal en pacientes con adenocarcinoma de próstata y comorbilidades previas. Sin embargo, por la naturaleza observacional de este estudio, no se puede atribuir el aumento de la prevalencia de eventos cardiocerebrovasculares en el grupo de mayor comorbilidad al uso específico de los agonistas de la GnRh.

Diversas estrategias se han planteado para la clasificación de la comorbilidad de los pacientes. El índice de Kaplan se desarrolla específicamente para la investigación en diabetes y clasifica la comorbilidad en vascular (hipertensión, cardiopatía, enfermedad vascular periférica, retinopatía y cerebrovascular) y no vascular (el pulmón, el hígado, los huesos, el riñón), dando puntuaciones del 0 al 3 dependiendo de la severidad de la afectación. La puntuación final es la de la patología con mayor severidad. Y existen dos adaptaciones: el Modified Medical Comorbidity Index y el Adult Comorbidity Evaluation 27 (ACE27). ${ }^{15}$ El índice de comorbilidades ACE27 es el instrumento de estudio de comorbilidad utilizado por el registro cancerológico del Barnes-Jewish Hospital en Estados Unidos. Él fue desarrollado a partir del índice de comorbilidad de Kaplan-Feinstein, en el cual se clasifica cada comorbilidad del paciente en leve o grado 1 , moderada o grado 2 y severa o grado 3 .

En este trabajo, el ACE-27 fue validado como una escala de comorbilidad basada en los registros médicos, lo cual implica un posible sesgo de información. Es así que se establece una de las debilidades del trabajo.

Los resultados de este estudio, generan la hipótesis de la posible asociación existente entre eventos cardiocerebrovasculares, el uso de análogos de GnRH y el grupo de riesgo cardiovascular. Otros diseños de estudio posteriores pueden ayudar a establecer la relación de causalidad.

\section{Conclusiones}

La prevalencia de eventos cardiocerebrovasculares fue del $7,5 \%$, en la población de estudio.

El aumento del grado de riesgo cardiovascular, según el índice ACE-27, se asoció con una mayor prevalencia de eventos cardiocerebrovasculares.

Se determinó una prevalencia del $25 \%$ de eventos cardiocerebrovasculares en el grupo de alto riesgo ACE27, expuesto a análogos de GnRh.

\section{Declaración}

No existen conflictos de intereses.

\section{Responsabilidades Éticas}

Protección de personas y animales. Los autores declaran que para esta investigación no se han realizado experimentos en seres humanos ni en animales.

Confidencialidad de los datos. Los autores declaran que han seguido los protocolos de su centro de trabajo sobre la publicación de datos de pacientes.

Derecho a la privacidad y consentimiento informado. Los autores declaran que en este artículo no aparecen datos de pacientes. 


\section{Bibliografía}

1 O'Farrell S, Sandström K, Garmo H, et al. Risk of thromboembolic disease in men with prostate cancer undergoing androgen deprivation therapy. BJU Int 2016;118(03):391-398

2 Keating NL, O'Malley AJ, Smith MR. Diabetes and cardiovascular disease during androgen deprivation therapy for prostate cancer. J Clin Oncol 2006;24(27):4448-4456

3 D'Amico AV, Denham JW, Crook J, et al. Influence of androgen suppression therapy for prostate cancer on the frequency and timing of fatal myocardial infarctions. J Clin Oncol 2007;25(17): $2420-2425$

4 Saigal CS, Gore JL, Krupski TL, Hanley J, Schonlau M, Litwin MS; Urologic Diseases in America Project. Androgen deprivation therapy increases cardiovascular morbidity in men with prostate cancer. Cancer 2007;110(07):1493-1500

5 Uttley L, Whyte S, Gomersall T, et al. Degarelix for Treating Advanced Hormone-Dependent Prostate Cancer: An Evidence Review Group Perspective of a NICE Single Technology Appraisal. Pharmacoeconomics 2017;35(07):717-726

6 Nanda A, Chen MH, Braccioforte MH, Moran BJ, D'Amico AV. Hormonal therapy use for prostate cancer and mortality in men with coronary artery disease-induced congestive heart failure or myocardial infarction. JAMA 2009;302(08):866-873

7 Mathers CD, Loncar D. Projections of global mortality and burden of disease from 2002 to 2030. PLoS Med 2006;3(11):e442

8 Alan J. Wein M, PhD(Hon), FACS, Louis R. Kavoussi, MD, MBA, Alan W. Partin, MD, PhD and Craig A. Peters, MD. Campbell-Walsh Urology, 11th Edition. 11 th ed. Elsevier, editor: Elsevier ed; 2016. 4168 p.

9 Siegel RL, Fedewa SA, Miller KD, et al. Cancer statistics for Hispanics/Latinos, 2015. CA Cancer J Clin 2015;65(06):457-480
10 Gandaglia G, Sun M, Popa I, et al. The impact of androgen-deprivation therapy (ADT) on the risk of cardiovascular (CV) events in patients with non-metastatic prostate cancer: a population-based study. BJU Int 2014;114(6b):E82-E89

11 Albertsen PC, Klotz L, Tombal B, Grady J, Olesen TK, Nilsson J. Cardiovascular morbidity associated with gonadotropin releasing hormone agonists and an antagonist. Eur Urol 2014;65(03): 565-573

12 Nguyen PL, Alibhai SM, Basaria S, et al. Adverse effects of androgen deprivation therapy and strategies to mitigate them. Eur Urol 2015;67(05):825-836

13 Carneiro A, Sasse AD, Wagner AA, et al. Cardiovascular events associated with androgen deprivation therapy in patients with prostate cancer: a systematic review and meta-analysis. World J Urol 2015;33(09):1281-1289

14 Sciarra A, Fasulo A, Ciardi A, et al. A meta-analysis and systematic review of randomized controlled trials with degarelix versus gonadotropin-releasing hormone agonists for advanced prostate cancer. Medicine (Baltimore) 2016;95(27):e3845

15 Abizanda Soler P, Paterna Mellinas G, Martínez Sánchez E, López Jiménez E. Evaluación de la comorbilidad en la población anciana: utilidad y validez de los instrumentos de medida. Rev Esp Geriatr Gerontol 2010;45(04):219-228

16 Gandaglia G, Sun M, Popa I. Cardiovascular mortality in patients with metastatic prostate cancer exposed to androgen deprivation therapy; a population based study. Clinical genitourinary cancer: Elsevier Inc; 2015:123-130

17 D’Amico AV, Chen MH, Renshaw AA, Loffredo M, Kantoff PW. Androgen suppression and radiation vs radiation alone for prostate cancer: a randomized trial. JAMA 2008;299(03):289-295 